\title{
Algunos aspectos de la dinámica poblacional del jurel Caranx hippos (pisces: carangidae) en Bocas de Ceniza, Caribe colombiano
}

\section{Some aspects of the population dynamics of the crevalle jack Caranx hippos (pisces: carangidae) in Bocas de Ceniza, colombian Caribbean}

Itala Caiafa $\mathrm{H}^{1}{ }^{1}$ Biol, Juan Narváez B, ${ }^{2 *}$ M.Sc, Silvana Borrero $\mathrm{F}^{3}$ Biol.

\begin{abstract}
${ }^{1}$ Ministerio de Agricultura y Desarrollo Rural, Bogotá, Colombia. ${ }^{2}$ Universidad del Magdalena, Programa de Ingeniería Pesquera, Grupo de Biodiversidad y Ecología Aplicada, Santa Marta, Magdalena, Colombia. ${ }^{3}$ Corporación Colombia Internacional (CCI), Barranquilla, Atlántico, Colombia. *Correspondencia: jcnarvaezb@yahoo.es
\end{abstract}

Recibido: Septiembre de 2009; Aceptado: Julio de 2010.

\section{RESUMEN}

Objetivo. Este estudio tuvo como propósito evaluar aspectos biológicos y la dinámica poblacional del Jurel (Caranx hippos) en el sector de Bocas de Ceniza, Caribe colombiano. Materiales y métodos. Entre Junio de 2005 y Junio de 2006, se recolectaron datos de frecuencias de longitudes comerciales para determinar la relación longitud horquilla-peso ( $\mathrm{LH}-\mathrm{W})$, la talla media de madurez sexual $\left(\mathrm{L}_{50 \%}\right)$, los parámetros de crecimiento $\left(\mathrm{K}, \mathrm{L}_{\infty} \mathrm{y}\right.$ $\mathrm{t}_{0}$ ), las tasas de mortalidad ( $\mathrm{M}, \mathrm{F}$ y $\mathrm{Z}$ ) y de explotación (E). Se contó con 1151 individuos, de los cuales 264 se usaron para el análisis biológico. Resultados. La relación LH-W difirió entre sexos. Se estimó una $L_{50 \%}$ en $63.6 \mathrm{~cm}$ de $\mathrm{LH}$. Los parámetros de crecimiento presentaron los siguientes valores: $L_{\infty}=91 \mathrm{~cm}$ de $L H, K=0.38$ año-1 y $t_{0}=0.32$ año. El valor del índice de desempeño tuvo un rango entre 3.33 y 3.73. La tasa de mortalidad total (Z) fue de 1.85 año-1 $^{-1}$ la mortalidad por pesca ( $F$ ) de 0.72 año-1 y la natural (M) en $1.13 \mathrm{año}^{-1}$. El estado de explotación (E) fue de $0.30 \mathrm{año}^{-1}$. Conclusiones. Según la tasa de explotación, el Jurel no presenta sobreexplotación en Bocas de Ceniza. Sin embargo, al comparar la talla media de captura con la $\mathrm{L}_{50 \%}$, la especie está siendo capturada con los principales artes de pesca antes de que el $50 \%$ de la población logre madurar. Se proponen pautas para el diseño de un sistema de manejo pesquero que garantice la conservación y el aprovechamiento sostenible de este recurso.

Palabras clave: Pesca, reproducción, Caranx hippos, Caribe, Colombia. (Fuente: AIMS) 


\section{ABSTRACT}

Objetive. To evaluate the biological aspects and the population dynamics of the Crevalle jack (Caranx hippos) from Bocas de Ceniza, colombian Caribbean. Materials and methods. Between June 2005 and June 2006 length frequency data were collected to determine fork length-weight relationship, mean maturity size $\left(\mathrm{L}_{50 \%}\right)$, Von Bertalanffy's equation growth parameters $\left(K, L_{\infty}\right.$ y $\left.t_{0}\right)$, mortality $(M, F$ y $Z$ ) and exploitation (E) rates. A total of 1151 individuals were sampled, and 264 were used for biologic analysis. Results. Fork lengthweight relationship showed differences between genders. $L_{50 \%}$ was estimated in $63.6 \mathrm{~cm}$ FL. Growth parameters values were: $L_{\infty}=91 \mathrm{~cm} \mathrm{FL}, K=0.38$ year $^{-1}$ y $t_{0}=0.32$ year. The comparative growth index $\left(\Phi^{\prime}\right)$ values ranged from 3.33 to 3.73 . Total mortality rate $(Z)$ was estimated as 1.85 year $^{-1}$, fishing mortality $(F)$ as 0.72 year $^{-1}$ and natural mortality (M) as 1.13 year $^{-1}$. Exploitation rate (E) was determined as 0.31 year $^{-1}$. Conclusions. The exploitation rate indicated that Crevalle jack from Bocas de Ceniza was not overexploited; before $50 \%$ of the population reach its sexual maturity. We propose a framework for a fishery management system, assuring the conservation and suitable use of this resource.

Key words: Fishing, reproduction, Caranx hippos, Caribe, Colombia. (Source: AIMS)

\section{INTRODUCCIÓN}

La familia Carangidae es uno de los grupos de peces de mayor importancia económica en las pesquerías tropicales y subtropicales del mundo $(1,2)$. De los desembarcos provenientes de la pesca artesanal en el departamento del Atlántico, el Jurel Caranx hippos (Linneo 1766) es una de las especies de mayor demanda (3). Representa el $47 \%$ en las capturas desembarcadas anualmente y el $70 \%$ de los ingresos económicos de la población de pescadores, siendo capturada principalmente con trasmallos $y$ en algunos casos con anzuelos y boliches (3). A pesar de esto, existe poca información detallada acerca de los aspectos biológicos y poblacionales que permitan dilucidar el estado de explotación pesquera en el que se encuentra su población en la desembocadura del río Magdalena.

Las estimaciones de los parámetros de crecimiento, las tasas de mortalidad y de explotación son parte importante en el estudio de la biología de los peces de interés comercial, ya que permiten determinar los niveles de explotación y a su vez proporcionan herramientas para la implementación de políticas de administración y control para un adecuado aprovechamiento (4-6). El objetivo de este trabajo fue determinar la relación LHW, la talla media de madurez sexual, los parámetros de crecimiento, las tasas de mortalidad y de explotación de C. hippos en Bocas de Ceniza para conocer el estado de explotación en este sector y fundamentar las bases científicas para proponer pautas orientadas al diseño de sistemas de manejo pesquero que conduzcan a una explotación sostenible de este importante recurso.

\section{MATERIALES Y MÉTODOS}

Área de estudio. Comprendió el estuario del río Magdalena en el Mar Caribe, sector

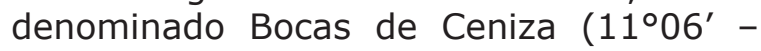
$11^{\circ} 08^{\prime}$ Norte; 7450' - 74²5' Oeste; Figura 1). Este sector se encuentra localizado al norte del departamento del Atlántico (3). El clima se caracteriza por la presencia de dos períodos climáticos que afectan la actividad de los pescadores (7); el seco que comienza a principios de diciembre y termina a finales de abril sin precipitaciones y una disminución del caudal del río Magdalena con un promedio de $4068 \mathrm{~m}^{3} \mathrm{~s}^{-1}(7,8)$. El período Iluvioso 


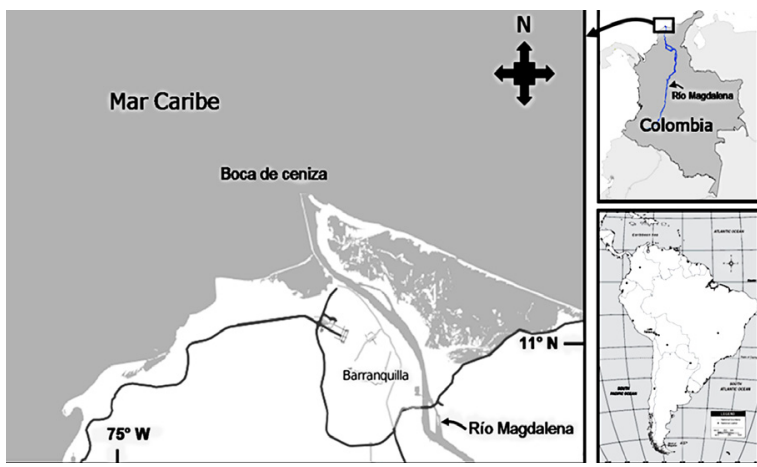

Figura 1. Mapa del área de estudio el área. Bocas de Ceniza. Escala 1: 100.000.

inicia en mayo y finaliza a principios de diciembre con disminución de los vientos y alta nubosidad $(7,9)$ y es cuando se presentan las mayores descargas de agua con un promedio de $10287 \mathrm{~m}^{3} \mathrm{~s}^{-1}(8)$.

Bocas de Ceniza es un estuario de cuña salina, donde su funcionamiento responde a la interacción entre la onda de marea y el aporte fluvial dentro de los contornos impuestos por la geometría del mismo estuario (9). El sector está influido por dos corrientes: la proveniente del Caribe con sentido oeste y la corriente de Panamá con dirección este (9). En esta área, la actividad pesquera es de tipo artesanal y cuenta con cerca de 250 pescadores que operan los artes de pesca trasmallos, anzuelos, palangres y boliche. Las capturas están representadas por especies marinas, así como de especies estuarinas. Se destacan Macrodon ancyclodon, Caranx crysos, C. hippos, Scomberomorus brasiliensis, Ariopsis sp., Bagre spp. y Arius props (3).

Fase de campo y laboratorio. Entre junio de 2005 y junio de 2006 se realizaron muestreos durante cinco días de cada mes para registrar frecuencias de longitudes de las capturas comerciales desembarcadas en el barrio Las Flores, Barranquilla. Las capturas fueron realizadas con anzuelos de calibre 7-10 y con boliches y trasmallos entre 8.89 y $20.32 \mathrm{~cm}$ de tamaño de malla. Cada individuo fue sexado y pesado (sin eviscerar) con una balanza de precisión de $0.1 \mathrm{~g}$. Dado que no todos los peces llegaron con las aletas caudales completas, se usó la longitud horquilla. Las mediciones se realizaron al límite inferior, utilizando un ictiómetro con $0.1 \mathrm{~cm}$ de precisión (3). Para el análisis biológico se realizaron observaciones macroscópicas de las gónadas para determinar el sexo y estado de maduración gonadal, tomando como referencia la clasificación cualitativa de Vazzoler (10), la cual consiste en cuatro estados: indiferenciados; inmaduros (I); en maduración (II); maduros (III) y desovados (IV).

Relación longitud-peso (LH-W). Se determinó la relación longitud-peso por sexo a través de la ecuación potencial $\mathrm{W}=\mathrm{aLH} b$, donde $\mathrm{W}$ es el peso total, $\mathrm{a}$ y $b$ son constantes de la regresión potencial y LH es la longitud horquilla. Para determinar diferencias en la relación $\mathrm{LH}-\mathrm{W}$ entre sexos, se realizó un análisis de covarianza (ANCOVA), donde la variable dependiente fue $W$, la variable independiente fue $\mathrm{LH}$ y el factor el sexo. Previo al análisis se evaluaron los supuestos de normalidad de los residuos (prueba de KolmogorovSmirnov), homogeneidad de varianzas (prueba de Bartlett), relación lineal entre la variable W y LH (análisis de correlación), y homogeneidad de las pendientes de la relación entre $\mathrm{W}$ y $\mathrm{LH}$.

Talla media de madurez sexual $\left(L_{50 \%}\right)$, composición de la captura por tallas y talla media de captura (TMC). Para evaluar el impacto de la pesquería sobre la biología de C. hippos, se calculó $\mathrm{L}_{50 \%}$. Se consideraron aquellos ejemplares cuyas gónadas se observaron en los estados III y IV. El cálculo se basó en el modelo logístico que relaciona la proporción de individuos maduros $[P(L)]$ con la longitud $(L ; 5)$ :

$$
P(L)=\frac{\beta}{1+\exp \left(\alpha_{1}-\alpha_{2} L\right)}
$$

donde, $\beta=1 ; \alpha 1$ y $\alpha 2$ son los parámetros de la regresión.

Para estimar $L_{50 \%}$ se usó la relación $-\alpha_{1} / \alpha_{2}$. Para ajustar el modelo se utilizó el método de regresión no lineal por mínimos cuadrados, usando el algoritmo iterativo de Newton. 
La composición de la captura por tallas fue usada para calcular la TMC y los parámetros de crecimiento. Los datos fueron agrupados mensualmente en marcas de clase cada dos centímetros. La relación entre la frecuencia relativa y el número total de individuos se empleó para calcular la TMC usando el procedimiento propuesto por Sparre y Venema (5); posteriormente, se calculó una TMC para cada arte de pesca observado. Las TMC fueron comparadas con la $L_{50 \%}$ para evaluar el impacto de la pesquería sobre la biología de la especie.

Parámetros de crecimiento. Los datos de longitud fueron agrupados mensualmente en marcas de clase de $1 \mathrm{~cm}$. Los parámetros de crecimiento se estimaron mediante tres rutinas contenidas en el paquete computacional FISAT versión 1.1 (4): el análisis de frecuencia electrónica (ELEFAN I), el de composición de longitudes de Shepherd (SLCA; 11) y el método de proyección de matrices (PROJMAT; 12). La rutina ELEFAN I trabaja con la ecuación de crecimiento estacionalizada de von Bertalanffy (ELEFAN I), siendo la talla expresada en función de la edad (5):

$L_{t}=L_{\infty}\left(1-e^{\left[-K\left(t-t_{0}\right)+(K C / 2 \pi) \operatorname{sen} 2 \pi\left(t-t_{2}\right)-(K C / 2 \pi) \operatorname{sen} 2 \pi\left(t_{0}-t_{s}\right)\right]}\right)$

donde, $L_{t}$ es la longitud a la edad $t ; L_{\infty}$ la longitud asintótica; $\mathrm{K}$ es la constante de crecimiento, $t_{0}$ es el parámetro de condición inicial, el cual determina el punto hipotético en el tiempo en el que el pez tiene una talla igual a cero; C es el parámetro de amplitud de la oscilación del crecimiento; ts es la parte del año en que la tasa de crecimiento es mínima.

La rutina SLCA estima el crecimiento independiente del número y posición de las modas en la muestra, basado en el ajuste de la función coseno:

$$
T_{(1)}=\frac{\operatorname{Sen} \pi\left(t_{\text {max }}-t_{\text {min }}\right)}{\pi\left(t_{\text {max }}-t_{\text {min }}\right)} \operatorname{Cos} 2 \pi\left(\bar{t}-t_{s}\right)
$$

donde, $\mathrm{t}_{\text {máx }} \mathrm{y} \mathrm{t}_{\text {mín }}$ son edades correspondientes a los límites superior e inferior de un determinado intervalo de clase; es la edad promedio $\mathrm{y}_{\mathrm{s}}$ es la fracción del año en la que fue tomada la muestra (6).
El PROJMAT proyecta una muestra de distribución de frecuencias de longitudes de tiempo $t$ a tiempo $t+1$, usando los valores de $\mathrm{K}$ y $\mathrm{L}_{\infty}$. Las frecuencias proyectadas fueron comparadas con las observadas por la suma de las diferencias de cuadrados, repitiendo el proceso hasta obtener los mejores estimativos (6).

Teniendo en cuenta que no es posible obtener una estimación de $t_{0}$ a partir de las frecuencias de longitudes $(13,14)$, este parámetro fue estimado a partir de la ecuación empírica de Pauly (15):

$$
\log _{0}\left(t_{0}\right)=-0.3922-\left[0.2752 \cdot \log _{0}\left(L_{\infty}\right)\right]-\left[1.038 \cdot \log _{0}(K)\right]
$$

donde, $t_{0}$ es la edad hipotética a la que comienza a crecer el animal, $L_{\infty}$ la longitud asintótica y $\mathrm{K}$ es la constante de crecimiento.

Para comparar las estimaciones de crecimiento entre métodos, se utilizó el índice de desempeño de crecimiento $\Phi^{\prime}$ (16). Adicionalmente, estos valores fueron comparados con las estimaciones de otras poblaciones de $C$. hippos documentados en la literatura. Este índice se estimó a partir de la expresión:

$\phi^{\prime}=\log _{0} K+2 \cdot \log _{0} L_{\infty}$

donde, $\mathrm{K}$ y $\mathrm{L}_{\infty}$ son los parámetros de crecimiento.

Dado que el valor estimado de $L_{\infty}$ en este estudio se basó en LH y los documentados en la literatura en longitud total (LT), para propósitos de realizar comparaciones entre los valores, se hizo una regresión lineal simple entre LT (dependiente) y LH (independiente) para hacer la conversión de LH a LT. Para esto se obtuvo el siguiente modelo lineal con datos de este estudio:

$L T=-0.4387+(1.2335 * \mathrm{LH})$

A partir de las frecuencias de LH de las capturas comerciales, se procedió a estimar las tasas de mortalidad total ( $Z$ ), natural (M) y por pesca (F) de la población de C. hippos. Z se estimó a partir de la curva de captura linealizada convertida a tallas (17): 
$\operatorname{Ln}\left(\frac{N_{i}}{\Delta t_{i}}\right)=a+b t_{i}^{\prime}$

donde, $N_{i}$ es el número de individuos capturados pertenecientes a la clase de longitud $\mathrm{i} ; \mathrm{t}^{\prime}$, es la edad relativa de la clase i; $\Delta t_{i}$ es el tiempo medio requerido por individuo para su crecimiento a través de la clase i.

M fue estimada usando la ecuación empírica de Pauly (17):

$\log _{0} M=0.0066-\left[0.279 \log _{0}\left(L_{\infty}\right)\right]+\left[0.6543 \log _{0}(K)\right]+\left[0.4634 \log _{0}(T)\right]$

donde, $L_{\infty}$ y $K$ son los parámetros de la ecuación de von Bertalanffy y $\mathrm{T}$ es la temperatura media anual del agua costera superficial en el área de estudio, la cual se consideró en $28.2^{\circ} \mathrm{C}$.

La tasa de mortalidad por pesca y la tasa de explotación fueron estimadas a partir de:

$$
F=Z-M \quad E=F / Z
$$

Para realizar las estimaciones de los parámetros se asumió un crecimiento estacionalizado de C. hippos, dado que a través del año suceden episodios ambientales en el área de estudio que podrían estar influyendo en el crecimiento de los peces. Tal es el caso de las condiciones estacionales del caudal del río Magdalena, las cuales pueden influir en las condiciones físicas, químicas y biológicas en su desembocadura (8). A esto se le suma, la influencia de los vientos Alisios en el sector de Bocas de Ceniza, puesto que cuando están en marcha y según su intensidad, también cambian las condiciones oceanográficas del sector (18). De igual forma, los conocimientos biológicos de la especie indican que $C$. hippos es una especie migratoria (1), a partir de lo cual también se puede inferir estacionalidad en el crecimiento. Con base a lo anterior se seleccionaron los valores hallados por ELEFAN I para estimar las tasas de mortalidad de $C$. hippos en este estudio.

Con el propósito de cuantificar el efecto de las muestras mensuales en los parámetros de crecimiento y obtener los valores de precisión de los mismos, se aplicó la técnica de remuestreo Jackknife (6). Esta consiste en estimar las $n$ muestras mensuales de frecuencia de tallas y secuencialmente son omitidas para obtener $\mathrm{n}$ combinaciones de cada uno de los parámetros. Para precisar esta estimación se usó el Error Porcentual (EP) y el Coeficiente de Variación (CV), utilizando las siguientes fórmulas:

$$
E P=\frac{\left|S t-S t_{j}\right| \cdot 100}{S t_{j}} \quad C V=\sqrt{\frac{\sum\left(S t_{i-1}-S t\right)^{2} / n(n-1)}{S t_{j}}} \cdot 100
$$

donde, St es el parámetro de crecimiento estimado usando la base de datos anual completa y $\mathrm{St}_{\mathrm{j}}$ es el estimador promedio derivado de las estimaciones sucesivas de Jackknife; $\mathrm{St}_{\mathrm{i}-1}$ es el parámetro estimado al omitir el mes i, y $n$ es el tamaño de la muestra anual $(n=12)$.

\section{RESULTADOS}

Durante el periodo de estudio se recolectaron 1151 individuos, de los cuales 868 fueron capturados con trasmallo, 120 con anzuelo y 167 con boliche; presentando un rango de talla comprendido entre 10.5 y $96.5 \mathrm{~cm}$ de longitud horquilla. Para el análisis de los aspectos biológicos estudiados se contó con 264 individuos, siendo 180 machos y 84 hembras, con un rango de talla entre 25 y $96.5 \mathrm{~cm}$ de LH y un peso total entre 281.5 y $10500 \mathrm{~g}$.

Relación longitud-peso ( LH-W). El análisis reveló que entre sexo la relación LH-W difirió significativamente, observándose que las hembras pesan más que los machos a una misma longitud (ANCOVA: F1, $260=21.5$; $\mathrm{p}<0.05)$. Por lo tanto, la relación $\mathrm{LH}-\mathrm{W}$ para machos fue $W=0.060 \mathrm{LH} 2.709(p<0.05)$ y para hembras $W=0.039$ LH2.825 $(p<0.05)$ (Figura 2). Los valores de b resultantes de las regresiones para ambos sexos fueron significativamente menores de 3 , indicando un crecimiento minorante (prueba $t$; $\mathrm{p}>0.05 ; 15)$.

Composición por tallas, talla media de madurez sexual $\left(L_{50 \%}\right)$ y talla media de captura (TMC). De manera general, las tallas mínimas de captura se registraron en 
Caiafa - Algunos aspectos de la dinámica poblacional del jurel Caranx hippos
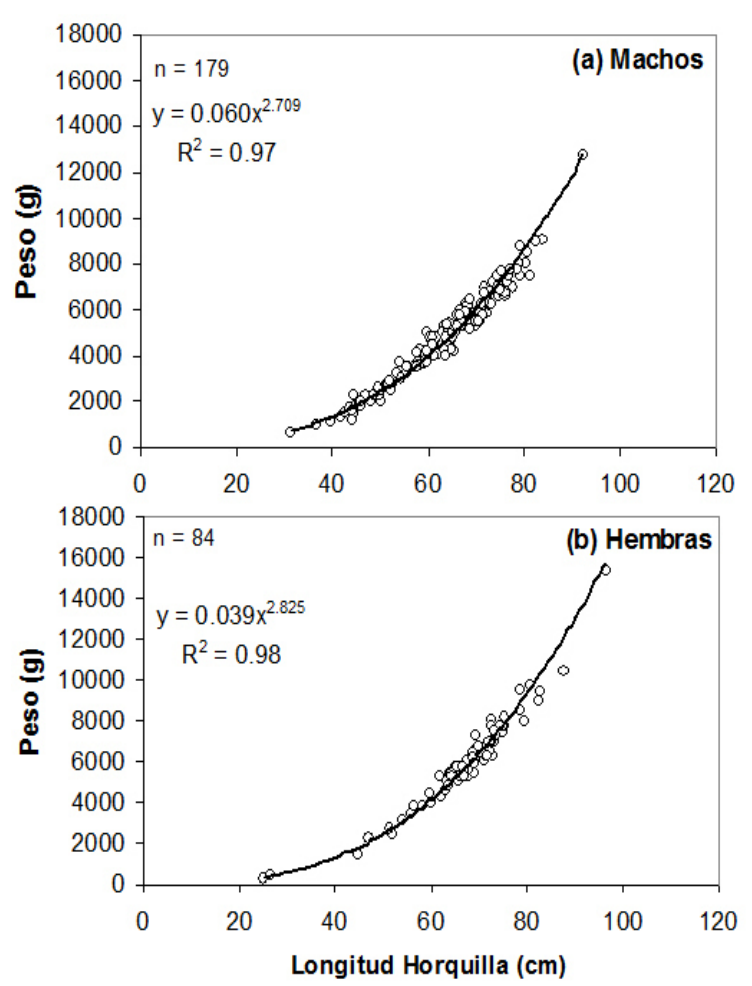

Figura 2. Relación LH - Peso para los individuos (a) machos y (b) hembras de $C$. hippos en Bocas de Ceniza, Caribe colombiano.

octubre, noviembre de 2005 y marzo, abril y junio de 2006; mientras que las máximas fueron frecuentes durante todo el periodo de estudio, excepto para octubre, enero y febrero (Figura 3).

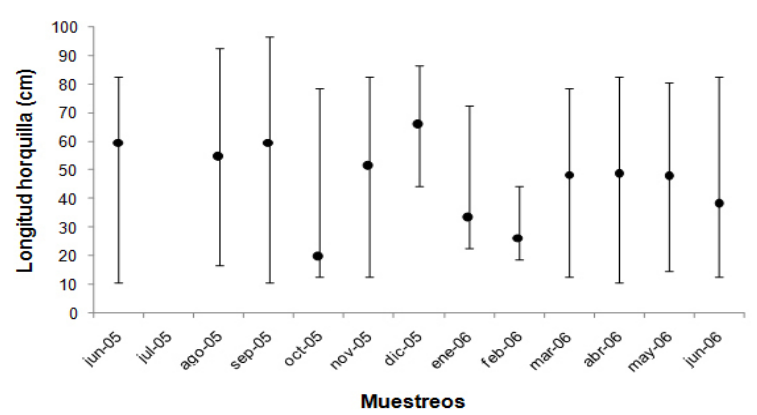

Figura 3. Tallas media de captura mensual de C. hippos en Bocas de Ceniza (junio de 2005 y junio de 2006). En las barras se indican los valores máximos y mínimos.

La $L_{50 \%}$ para sexos combinados fue $63.6 \mathrm{~cm}$ de LH (IC al 95\%: 59.0-68.4 cm). Para el caso de machos y hembras fueron $62.6 \mathrm{~cm}$ (IC al 95\%: $58.3-70 \mathrm{~cm}$ ) y $66.2 \mathrm{~cm}$ (IC al 95\%: 56.1-76.2 cm), respectivamente (Figura 4). La TMC global fue estimada en $50.3 \mathrm{~cm}$ de LH. Para el caso de trasmallos, anzuelos y boliches se estimaron en 56.0, 56.2 y $17.8 \mathrm{~cm}$, respectivamente (Figura $5 a, 5 b$ y $5 c)$, por debajo de la $L_{50 \%}$.

Crecimiento. Los resultados de $L_{\infty}$ y $K$ proporcionados por los métodos ELEFAN I, SLCA y PROJMAT son presentados en la tabla 1. Se observa que estos tres métodos suministraron diferentes valores para cada uno de los parámetros de crecimiento. El análisis de Jackknife mostró que existe efecto de los datos sobre los diferentes

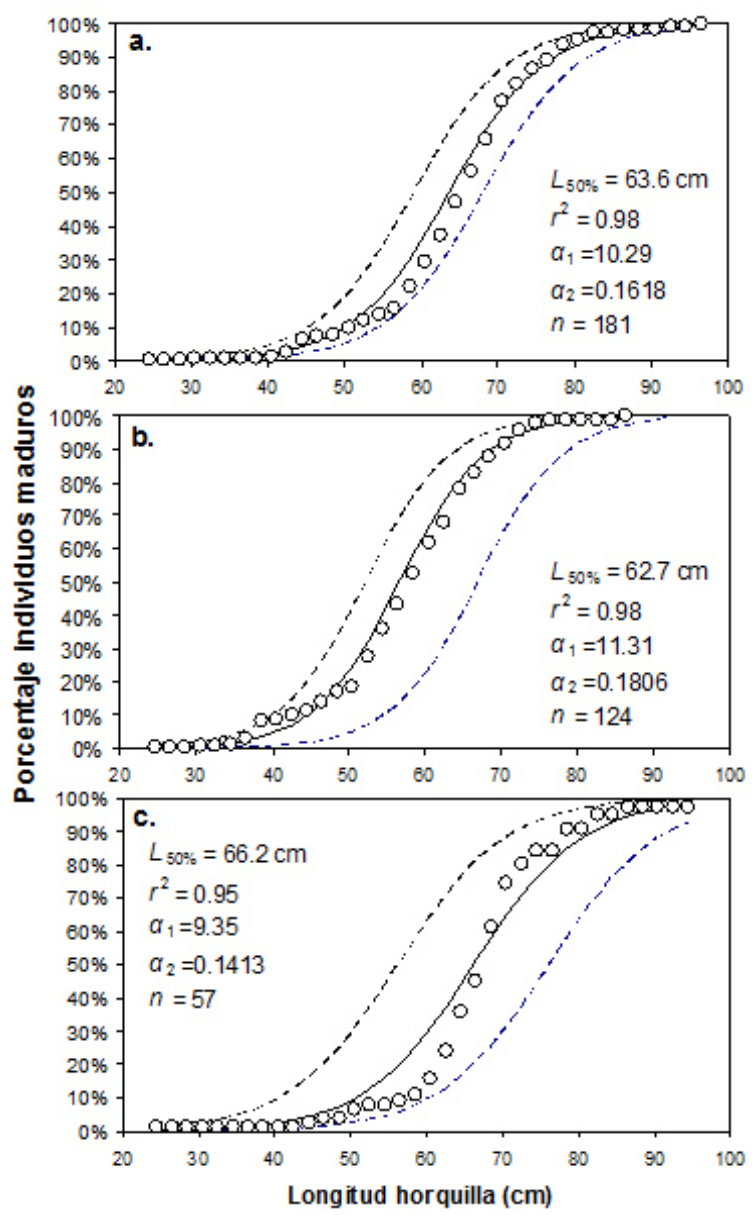

Figura 4. Curva de madurez sexual de $C$. hippos en Bocas de Ceniza para (a) sexos combinados, (b) machos y (c) hembras. Los puntos indican los datos observados, la línea continua es el modelo ajustado y las punteadas son los límites inferior y superior del intervalo de confianza (95\%). Los parámetros fueron significativos $(p<0.05)$. 

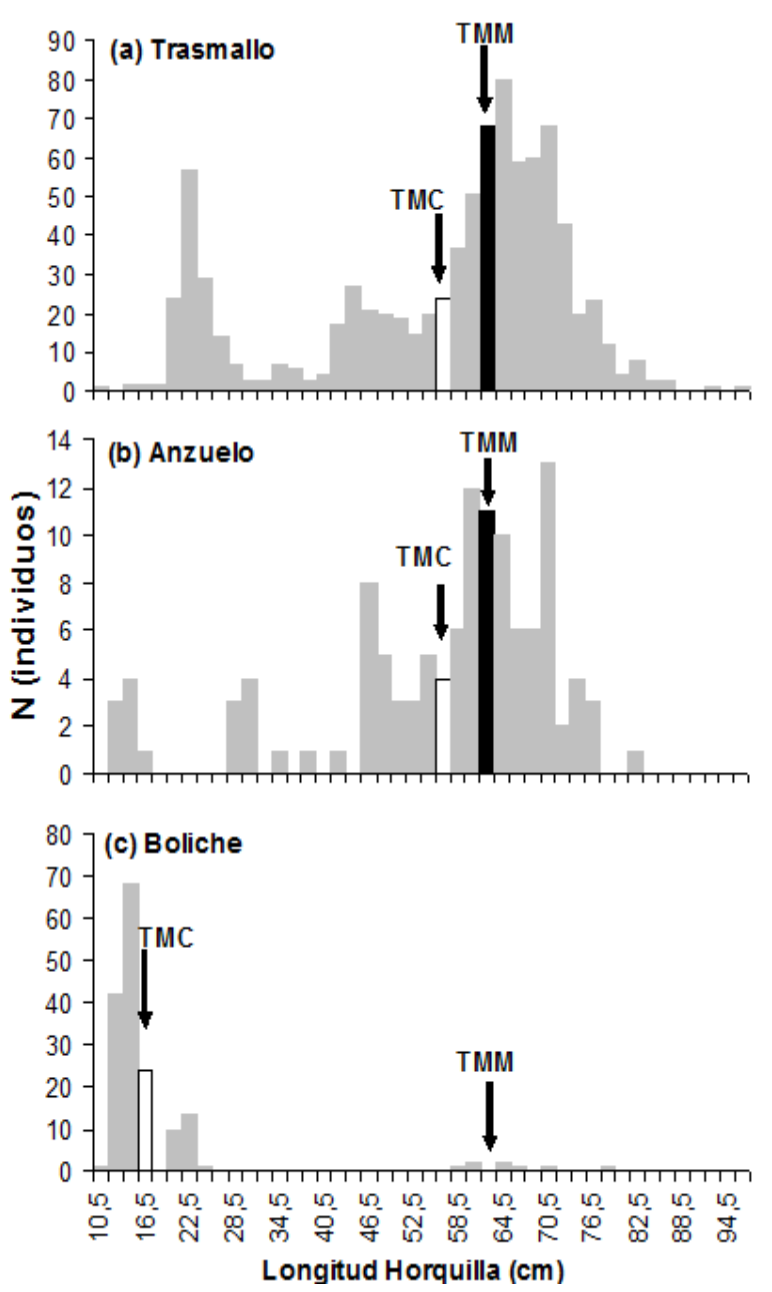

Figura 5. Composición de tallas medias de captura para C. hippos en Bocas de Ceniza. TMC =Talla media de captura; TMM=Talla media de madurez sexual.

parámetros de crecimiento, especialmente para SLCA y PROJMAT. Para el caso de ELEFAN I, $L_{\infty}$ varió de 77.4 a $100 \mathrm{~cm}$, mientras que los valores de $\mathrm{K}$ estuvieron entre 0.20 y 0.75 año $^{-1}$ (Figura 6). Con SLCA se obtuvieron los valores más altos de $L_{\infty}$ y $K$ con valores entre 78.25 y $120 \mathrm{~cm}$ y entre 0.3 y 1.0 año $^{-1}$, respectivamente. De acuerdo con los valores arrojados por PROJMAT, se observó menor dispersión para $\mathrm{K}$ fluctuando entre 0.10 y $0.17 \mathrm{año}^{-1}$. Lo contrario ocurrió para $\mathrm{L}_{\infty}$, donde los valores variaron entre 87 y $120 \mathrm{~cm}$, siendo este último mayor al observado en los estimadores de crecimiento anteriores. Los valores de $\mathrm{K}$ estimados mostraron diferencias entre métodos, siendo los más altos los calculados por ELEFAN I y SLCA (Tabla 1). El valor obtenido para la edad hipotética
Tabla 1. Parámetros de crecimiento de $C$. hippos en Bocas de Ceniza a partir de tres métodos, ELEFAN I, SLCA, PROJMAT y la ecuación empírica de Pauly para $t_{0}$. $G$ son los valores estimados a partir de los 12 meses de muestreo; $\mathrm{P}$ es el promedio de los datos generados por el análisis de Jackknife; PE es el porcentaje de error; CV es el coeficiente de variación.

\begin{tabular}{|c|c|c|c|c|}
\hline \multirow{2}{*}{ Parámetros } & \multicolumn{4}{|c|}{ Método } \\
\hline & & ELEFAN I & SLCA & PROJMAT \\
\hline \multirow[t]{4}{*}{$\mathrm{L} \infty(\mathrm{cm})$} & G & 91.00 & 98.33 & 105.47 \\
\hline & $P$ & 87.97 & 104.32 & 103.38 \\
\hline & PE (\%) & 3.44 & 5.74 & 2.02 \\
\hline & CV $(\%)$ & 2.44 & 3.81 & 2.59 \\
\hline \multirow[t]{4}{*}{$\mathrm{K}\left(\mathrm{año} 0^{-1}\right)$} & G & 0.38 & 0.31 & 0.1 \\
\hline & $P$ & 0.45 & 0.71 & 0.11 \\
\hline & PE (\%) & 19.53 & 56.12 & 6.90 \\
\hline & CV $(\%)$ & 11.43 & 16.93 & 15.49 \\
\hline \multirow[t]{4}{*}{$t_{0}$ (año) } & G & 0.32 & 0.39 & 1.227 \\
\hline & $P$ & 0.3 & 0.208 & 1.177 \\
\hline & PE (\%) & 10.028 & 84.85 & 4.286 \\
\hline & CV $(\%)$ & 15.13 & 24.75 & 6.34 \\
\hline$\Phi^{\prime}$ & & $3.33-3.73$ & $3.17-4.45$ & $2.76-3.34$ \\
\hline $\begin{array}{l}\text { Bondad de } \\
\text { ajuste }\end{array}$ & & $\mathrm{Rn}=0.33$ & $S=34.86$ & \\
\hline
\end{tabular}

a la que comienza a crecer el animal ( $t_{0}$ ) mediante la ecuación empírica de Pauly (20) fue de 0.32 año, con un coeficiente de variación de $13.92 \%$ y un porcentaje de error (PE) de 10.02. El análisis del índice de desempeño ǿ mostró valores similares entre ELEFAN I (3.33-3.73) y SLCA (3.174.45 ), lo que no ocurrió para PROJMAT que mostró valores inferiores a los anteriores (2.76-3.34). Aún así, la menor variación se presentó para ELEFAN I, seguido por PROJMAT y SLCA, siendo este último el que mayor rango presentó. Teniendo en cuenta el coeficiente de variación (CV) de cada uno de los parámetros, se encontró que los estimativos con ELEFAN I del análisis de Jackknife tendieron a proporcionar menores valores para $L_{\infty}$, a diferencia de SLCA y PROJMAT los cuales alcanzaron valores más altos (Tabla 1 ). El resultado del PE obtenido para $\mathrm{K}$ con PROJMAT fue menor al hallado para ELEFAN I y SLCA. De acuerdo con esto, el método que arrojó los estimativos más creíbles de los parámetros 
Caiafa - Algunos aspectos de la dinámica poblacional del jurel Caranx hippos
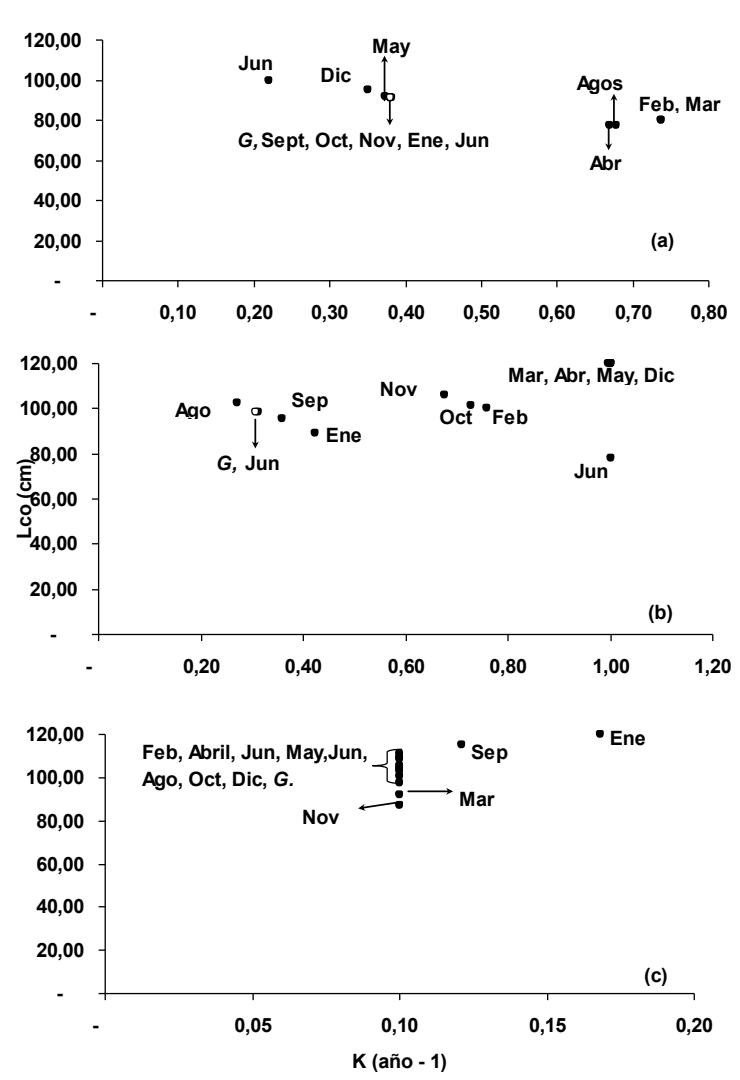

Figura 6. Relación entre $\mathrm{L}_{\infty}$ y $\mathrm{K}$ para $C$. hippos en Bocas de Ceniza, estimado a partir de: (a) ELEFAN I, (b) SLCA y (c) PROJMAT. Los puntos hacen referencia a los meses omitidos por el análisis de jackknife.

de crecimiento fue PROJMAT, seguido de ELEFAN I y por último SLCA.

Mortalidad y estado de explotación. Con base en los siguientes parámetros de crecimiento: $\mathrm{K}=0.38 \mathrm{año}^{-1} ; \mathrm{L}_{\infty}=91 \mathrm{~cm}$ $\mathrm{LH} ; \mathrm{t}_{\mathrm{o}}=0.32$ año; oscilación estacional en el patrón de crecimiento (C) de 0.5 y una tasa de crecimiento mínima (WP) de 0.3, se estimó una tasa de mortalidad total

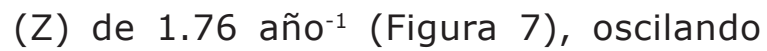
entre 1.38 y 2.22 año-1 (a partir del $^{-1}$ análisis de Jackknife).

La mortalidad natural (M) fue de 1.12 año-1, variando entre 1.10 y 1.14 año${ }^{1}$. La tasa de mortalidad por pesca (F) se estimó en 0.63 año $^{-1}$, presentando un rango de variación entre 0.2 y 1.06 $a n ̃ o^{-1}$. Los valores de la relación $F$ y $Z$ para estimar la tasa de explotación $(E)$, presentó un valor máximo de 0.5 año-1, $^{-1}$

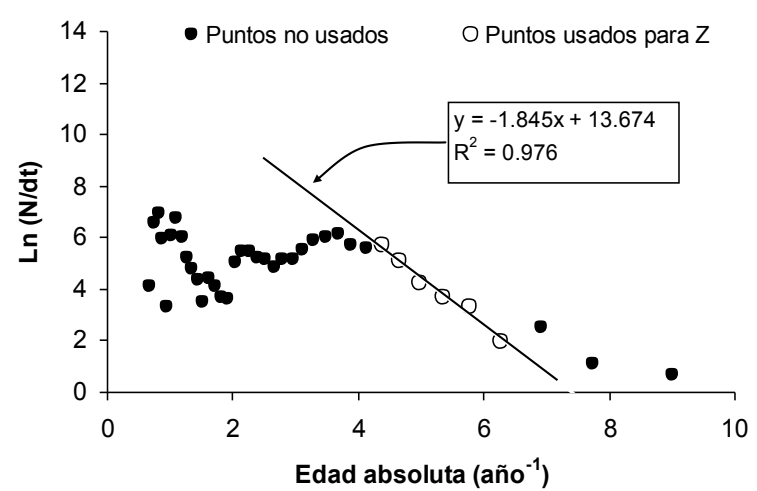

Figura 7. Curva de captura usada para la estimación de $Z$ para $C$. hippos en Bocas de Ceniza a partir de los 12 meses muestreados. La línea de regresión fue fijada para calcular $\mathbf{Z}$.

presentándose un promedio de $0.30 \mathrm{año}^{-1}$.

\section{DISCUSIÓN}

Relación longitud-peso (LH-W). El concepto de crecimiento implica cambios relativos en la talla y el peso de los individuos (19), y su relación tiene varios usos en la investigación pesquera. Por ejemplo, es útil en los modelos de análisis de stock pesqueros para estimar la biomasa de una población; para conocer la condición de un pez; para comparar la historia de vida de ciertas especies entre regiones; y para determinar el tipo de crecimiento que suelen presentar los peces (20). C. hippos, presenta un crecimiento en peso de tipo alométrico minorante $(<3 ; 15)$, indicando que presenta tasas de crecimiento diferencial para distintas partes del cuerpo, en donde la longitud aumenta a una tasa mayor que el peso.

Los resultados indican que esta condición fue más evidente para machos que para hembras, dado que estas últimas, a pesar de presentar un crecimiento alométrico minorante, se aproximan a tener un crecimiento isométrico. Esto fue concordante con el resultado de la ANCOVA, la cual indicó que a una misma LH existen diferencias en el peso entre sexos. Las hembras de esta especie presentan mayor peso y altura corporal que los machos. Por otro lado, es importante resaltar que debido a que las muestras fueron 
recolectadas durante un año, la relación LH-W en este estudio no representa una particular época del año. En este sentido, los valores de b podrían considerarse como un valor promedio anual para la especie (20). Los resultados de esta investigación fueron similares a los documentados para la especie en diferentes áreas geográficas. Por ejemplo, se documentaron valores de b para sexos combinados de 2.73 en el Sur de La Florida (21), de 2.85 en la India (1) y de 2.91 en el Golfo de Salamanca, Colombia (22).

Crecimiento, mortalidad y estado de explotación. Con base en los parámetros de crecimiento obtenidos por ELEFAN I, la longitud máxima estimada para $C$. hippos en Bocas de Ceniza fue de $91 \mathrm{~cm}$ LH que corresponde a $111.81 \mathrm{~cm}$ de LT. Si este último es convertido a edad corresponde a 14 años, alcanzada con una tasa de crecimiento $\mathrm{K}$ de $0.38 \mathrm{año}^{-1}$, sugiriendo que $C$. hippos es una especie longeva (17). Por otro lado, teniendo en cuenta la curva de crecimiento, esta especie creció rápidamente hasta la edad de 5 años. Este dato coincide para otros Carángidos, como el de Trachinotus falcatus, el cual alcanza una talla máxima de $90 \mathrm{~cm}$ y presenta también un crecimiento máximo de 5 años, a partir de la cual inicia la fase estacionaria de su crecimiento (2).

Por otra parte, los valores de $\mathrm{L}_{\infty}$ estimado para C. hippos en este estudio fueron superiores a los documentados en la literatura; mientras que los del índice de desempeño ǿ revisados en la literatura mostraron valores muy cercanos al estimado en este estudio (Tabla 2). Tomando este índice como un criterio de comparación entre los valores de los parámetros de crecimiento obtenidos y teniendo en cuenta que $C$. hippos es una especie migratoria (23), se puede suponer que el crecimiento de esta especie es concordante en el descrito en diferentes áreas de su distribución mundial (Tabla 2).

El patrón anual de crecimiento de $C$. hippos mostró oscilaciones $(C=0.5)$ con una tasa mínima de crecimiento en el mes de septiembre $(\mathrm{WP}=0.3)$. Este patrón puede
Tabla 2. Parámetros de crecimiento de $C$. hippos encontrados en la literatura y los estimados a partir de este estudio por medio de ELEFAN I. Los valores de ǿ con asteriscos fueron calculados en este estudio; los datos de $L_{\infty}$ con asterisco indican un valor medido a LT.

\begin{tabular}{lcccc}
\hline \multicolumn{1}{c}{ País } & $\mathrm{L}_{\infty}(\mathrm{cm})$ & $\mathrm{K}\left(\mathrm{año}{ }^{-1}\right)$ & \multicolumn{1}{c}{} & Fuente \\
\hline India & $49.80^{*}$ & 0.770 & 3.28 & Torres et al (29) \\
India & $44.40^{*}$ & 0.650 & 3.10 & Reuben et al (1) \\
Florida & $98.04^{*}$ & 0.217 & $3.32^{*}$ & Snelson (30) \\
Colombia & $91.00 \mathrm{LH}$ & 0.380 & 3.53 & Este estudio \\
& $111.81^{*}$ & & & \\
\hline
\end{tabular}

estar influido por uno de los aspectos más característicos en el crecimiento de los peces: la variabilidad observada entre individuos de una población y entre las poblaciones de una especie, la cual está directamente relacionada con el medio ambiente (24). Por ejemplo, la variabilidad en las condiciones físico-químicas del agua (24) y la calidad y cantidad de alimento (19) condicionan la migración de una especie, la cual abarca desplazamientos estaciónales entre las zonas de cría, alimentación y desove $(15,25)$.

Con base en lo anterior y teniendo en cuenta las frecuencias de longitudes observadas durante el periodo de estudio, las cuales también indican la posible presencia de estacionalidad en el crecimiento (13), se puede inferir que el crecimiento de $C$. hippos es estacional. Con respecto a la tasa mínima de crecimiento, este acontecimiento puede estar dado posiblemente por la etapa reproductiva de la especie, puesto que para septiembre se observó un incremento en el número de individuos maduros (en estadios III).

De otro lado, la relación entre la tasa de mortalidad por pesca (F) y mortalidad total (Z) de 0.3, indica que la población en el área de Bocas de Ceniza no está corriendo riesgo de ser sobreexplotada, bajo la premisa de que una población alcanza su rendimiento óptimo cuando la fracción de muertes causada por $\mathrm{F}$ está por encima de $0.5(6,13)$. Sin embargo, es importante resaltar que cuando $L_{50 \%}$ fue comparada con la TMC, C. hippos presentó una mayor presión sobre la población de adultos, 
frecuentemente capturada con trasmallo y anzuelo. La situación empeora con el uso del boliche, el cual no sólo afecta a los adultos, sino también a los jóvenes, lo que no permite la renovación natural de este recurso pesquero.

\section{Propuesta para el diseño de un sistema} de manejo pesquero. Partiendo de la base de que la sobrepesca puede tener lugar en tres formas diferentes: (i) en relación al crecimiento; (ii) con respecto al reclutamiento; y (iii) sobrepesca que afecta el ecosistema (15). En este sentido, la pesca de $C$. hippos en el sector de Bocas de Ceniza se puede clasificar dentro del primer ítem, la cual tiene lugar cuando los individuos son capturados antes de que alcancen la talla necesaria para garantizar la renovación biológica de la población. Lo anterior se ha convertido en un problema común para la mayoría de las especies comerciales, y viene siendo documentado para muchas especies costeras en el Caribe colombiano [por ejemplo: Ariopsis sp., Cathorops mapale, Eugerres plumieri, Mugil incilis $(6,26)]$.

Todo lo anterior, hace necesario que se inviertan esfuerzos para diseñar estrategias de manejo para $C$. hippos en la pesquería de Bocas de Ceniza, de tal manera que sean consistentes con el enfoque precautorio para la pesca responsable de este recurso (27). Caddy (27) sugiere que las estrategias deben ser aplicadas dentro de un marco de redundancia de manejo donde tal marco podría consistir en una mezcla de medidas que involucre criterios de selectividad de artes, vedas temporales y el aumento de las tallas mínimas de captura. En este sentido, tomando en cuenta la $\mathrm{L}_{50 \%}$ como un punto de referencia límite (28), aquí se recomienda que la talla mínima de captura de este recurso deba fijarse en $64 \mathrm{~cm}$ de longitud horquilla. Asimismo, en todos los artes de pesca utilizados se propone incrementar los tamaños de malla por encima de los $8.89 \mathrm{~cm}$ y usar calibres de anzuelos dirigidos a capturar peces más grandes. En las condiciones actuales estos artes de pesca están afectando directamente a la fracción de individuos jóvenes que ingresan a la primera fase de madurez sexual. Con las medidas anteriores, complementadas con otras variables como la captura, el esfuerzo de pesca y la captura por unidad de esfuerzo, así como también factores de sociales y económicos, se espera que $C$. hippos se capture en una mayor proporción de individuos por encima de la $\mathrm{L}_{50 \%}$ para garantizar la sostenibilidad de este recurso a través del tiempo. En este sentido, es importante tomar en cuenta la condición multiespecífica y multiflota de la pesquería y por lo tanto, se recomienda un proceso de concertación entre la comunidad de pescadores, los comerciantes y las entidades pertinentes para llegar a acuerdos dirigidos a una explotación responsable del recurso.

En conclusión, el Jurel no presenta sobreexplotación en Bocas de Ceniza. Sin embargo, al comparar la talla media de captura con la $L_{50 \%}$, la especie está siendo capturada con los principales artes de pesca antes de que el $50 \%$ de la población logre madurar. Se proponen pautas para el diseño de un sistema de manejo pesquero que garantice la conservación y el aprovechamiento sostenible de este recurso.

\section{Agradecimientos}

A la comunidad de pescadores, a los miembros de ASOPESCAR y COOPEZ. A los hermanos Villa y a la pescadería El Nuevo Tambó por su colaboración en la coordinación de actividades y suministro de información de campo. A los profesores G. Lozano, L.C. Gutiérrez, O. Galvis, J. Blanco y E. Viloria. 


\section{REFERENCIAS}

1. Reuben, S, HM Kasim, S Sivakami, PN Radhakrihnan, KN Kurup, M Sivadas, A Noble, KVS Nair, Raje SG. Fishery, biology and stock assessment of Carangid resources from the Indian seas. Indian J Fish 1992; 39(3,4):195-234.

2. Crabtree, R, PB Hood, Snodgrass D. Age, grouth and reproduction of permit (Trachinotus falcatus) in Florida waters. Fish Bull 2002; 100:26-34.

3. Rivera R, Solano, G. Diagnóstico de la actividad pesquera artesanal en la zona costera del departamento del Atlántico, Caribe colombiano. Barranquilla, Colombia: INCODER; 2005.

4. Gayalino, FC, P Sparre, Pauly D. The FISAT User Guide. FAO - ICLARM Stock Assessment Tools. Roma: FAO; 1993.

5. Sparre, P., Venema S. Introduccion a la evaluación de recursos pesqueros tropicales. Roma: FAO; 1995. Documento Técnico de Pesca: 306/1.

6. Rueda, M, Santos-Martínez A. Population dynamics of the striped mojarra Eugerres plumieri from the Ciénaga Grande de Santa Marta, Colombia. Fish Res 1999; 42:155-166.

7. Blanco, JA, EA Viloria, Narváez B JC. ENSO and interannual salinity changes in the Ciénaga Grande de Santa Marta coastal lagoon system, colombian Caribbean. Estuar Coast Shelf Sci 2006; 66:157-167.

8. Restrepo, JD, Kjerfve B. Magdalena river: interannual variability (19751995) and revised water discharge and sediment load estimates. J Hydrol 2000; 235(1-2):37-149.
9. Acevedo A, Gutiérrez ML. Algunos aspectos reproductivos del toyo Rhizotrionodon porosos (1831) (Elasmobranchii: Carcharinidae). En el sector de Bocas de Ceniza, Caribe colombiano. [Trabajo de pregrado en Biología]. Barranquilla, Colombia: Universidad del Atlántico, Facultad de Ciencias Básicas; 1999.

10. Vazzoler, EAM. Manual de metodos para estudos biologicos de populacoes de peixes: reproducao e crescimento. Brasília: CNPQ; 1982.

11. Sheperd, JG. A weakly parametric method for the analysis of length composition data. In: Pauly, D, Morgan GP editors. Length-based Methods in Fisheries Research. Manila, ICLARM Conference Proceedings 13. 1987.

12. Leslie, PH. The use of matrices in certain population mathematics. Biometrika 1945; 35:213-245.

13. Gulland, JA, Rosemberg AA. Exámen de los métodos que se basan en la talla para evaluar las poblaciones de peces. Roma: FAO; 1992.

14. Tordecilla-Petro G, S SánchezBanda, Olaya-Nieto $\mathrm{CH}$. Crecimiento y mortalidad del moncholo (Hoplias malabaricus) en la Ciénaga Grande de Lorica, Colombia. Rev MVZ Córdoba $2005 ; 10(2): 623-632$.

15. Pauly, D. Algunos métodos simples para la evaluación de recursos pesqueros tropicales. Roma: FAO; 1983.

16. Pauly, D, Munro JL. Once more on the comparison of growth in fish and invertebrates. Fishb 1984; 1:21. 
17. Pauly, D. Fish population dynamics in tropical waters; a manual for use with programmable calculators. ICLARM Stud Rev 1984; 8:235.

18. Blanco, JA. Las variaciones ambientales estacionales en las aguas costeras y su importancia para la pesca en la región de Santa Marta, Caribe Colombiano. [Tesis de maestría en Biología]. Santa Marta-Bogotá. Universidad Nacional de Colombia, Facultad de Ciencias Básicas; 1988.

19. Abaunza L, Karlou-Riga C, Murta A, Eltink W, García Santamaría MT, Zimmermman C, Hammer C, et al. Growth and reproduction of horse mackerel, Trachurus trachurus (Carangidae). Rev Fish Biol Fish 2003; 13:27-61.

20. Froese, R. Cubelaw, condition factorand weight-length relationships: history, meta-analysis and recommendations. J Appl Ichthyol 2006; 22:241-253.

21. Bohnsack, JA, Harper DE. Lengthweight relationships of selected marine reef fishes from the Southeastern United States and the Caribbean. NOAA Technical Memorandum; 1988. NMFS-SEFC-215.

22. Duarte, LO, CB García, I Moreno, G Melo, P Navajas, N Sandoval, et al. Atlas demográfico de los peces demersales del Golfo de Salamanca, Caribe colombiano: dinámica poblacional, distribución, alimentación y reproducción. [Libro digital en CD]. Versión 1. Santa Marta: INVEMARUNAL-COLCIENCIAS; 1999.
23. Honebrink, R. A review of the biology of the family carangidae whith emphasis on species found in Hawaiian waters. Honolulu: Division of acuatic resources; 2000.

24. Macer, CT. Some aspects of the biology of the horse mackerel (Trachurus trachurus (L)) in waters around Britain. J Fish Biol 1977; 10:51-62.

25. Harden, F R. Fish migration. London: Arnold; 1968.

26. Narváez B, JC, F Herrera, Blanco J. Efectos de los artes de pesca sobre el tamaño de los peces en una pesquería artesanal del Caribe colombiano. Bol Invest Mar Cost 2008; 37(2):163-187.

27. Caddy, JF. Fisheries management in the twenty-first century: will new paradigms apply?. Rev Fish Biol Fish 1999; 9:1-43.

28. Caddy, JF. Limit reference points, traffic lights, and holistic approaches to fisheries management with minimal stock assessment input. Fish Res 2002; 56(2):133-137.

29. Torres, F JR, MBTM Norizam, LR Garcés, Silvestre GT. Compilation of population parameters of fishes species commonly caught in trawls in south and southeast Asia. Manila: World fish center; 2001.

30. Snelson, FF. Biological studies of crevalle jack (Caranx hippos) in Florida. St. Petesburg: Florida Marine Reseach Institute; 1992. 